\title{
The XENON100 Dark Matter Experiment
}

\author{
Marc Schumann ${ }^{1}$, Eirini Tziaferi ${ }^{2}$ for the XENON100 collaboration \\ ${ }^{1}$ Rice University, 6100 Main St., Houston TX, USA \\ ${ }^{2}$ University of Zürich, Winterthurerstr. 190, Zürich, Switzerland
}

DOI: http://dx.doi.org/10.3204/DESY-PROC-2008-02/schumann_marc

The XENON collaboration searches for Dark Matter using a dual-phase liquid xenon (LXe) TPC to measure nuclear recoils from WIMPs scattered off Xe nuclei. The next step of the XENON program is XENON100, a $170 \mathrm{~kg}$ detector with a fiducial mass of about $50 \mathrm{~kg}$ surrounded by an active LXe veto. It is currently installed underground at LNGS (Italy). First test measurements have started, and it will be operational in fall 2008. This paper introduces XENON100, describes all major components, and focuses on the studies of the expected gamma and neutron background. All used materials have been screened to obtain their intrinsic radioactivity, which allows to predict the background and determines the final sensitivity.

\section{Introduction}

There is overwhelming observational evidence that about $25 \%$ of the universe consists of cold Dark Matter [1]. The nature of the Dark Matter particle, however, remains unknown. One of the best motivated candidates is the WIMP (weakly interacting massive particle) that arises naturally in particle physics theories beyond the Standard Model like Supersymmetry, Large Extra Dimensions, and Little Higgs [2].

The XENON Dark Matter experiment searches for nuclear recoils from WIMPs scattering elastically off xenon nuclei in a two-phase (liquid/gas) time projection chamber (TPC).

Xenon as Dark Matter target The expected WIMP interaction rate is $<0.1$ events $/ \mathrm{kg} /$ day and the nuclear recoils have energies $<50 \mathrm{keV}$, showing a steeply falling featureless spectrum. The noble gas xenon - an efficient and fast scintillator $(178 \mathrm{~nm})$ without any long lived isotopes is an ideal choice for the target material: its high mass number $A \sim 131$ enhances the interaction probability for spin-independent WIMP-nucleon interactions $\left(\sigma \propto A^{2}\right)$ and its high $Z=54$ and high density $\left(\rho=3 \mathrm{~g} / \mathrm{cm}^{3}\right)$ provide powerful self-shielding in a compact detector geometry.

XENON is a two-phase TPC with two arrays of photomultipliers (PMTs) above and below the target region [3]. The principle of operation is illustrated in Fig. 1: a particle interacting with the xenon generates primary scintillation light $(S 1)$. The electrons from ionization in the target are drifted upwards by a strong electric field $(\sim 1 \mathrm{kV} / \mathrm{cm})$ applied at the cathode, and are extracted to the gas phase (extraction field $\sim 10 \mathrm{kV} / \mathrm{cm}$ ) where they create secondary scintillation light $(S 2)$. The primary vertex can be reconstructed within $\sim 3 \mathrm{~mm}$ using the electron drift time and the hit pattern information on the PMT arrays.

Important information for an event-by-event discrimination of signal and background events is obtained from the ratio of secondary and primary scintillation light, $S 2 / S 1$. This ratio is much 


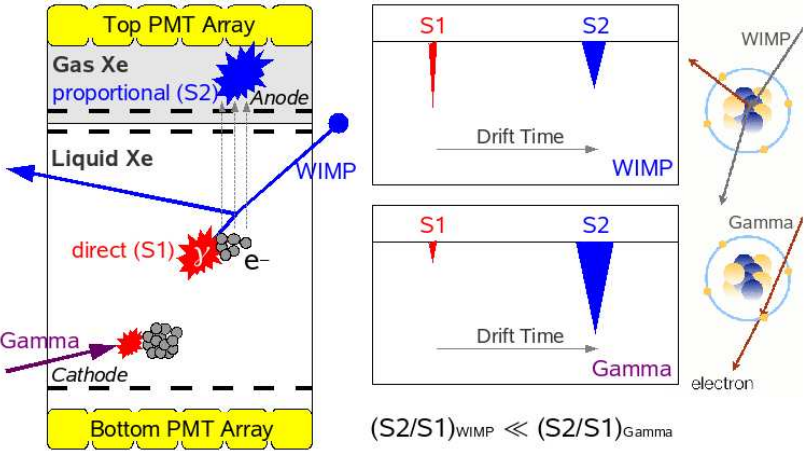

Figure 1: In a XENON TPC, primary $(S 1)$ and secondary scintillation light $(S 2)$ are different for electron recoil $(\beta, \gamma)$ and nuclear recoil-like events (WIMPs, neutrons), and their ratio $S 2 / S 1$ can be used for effective eventby-event discrimination. The event vertex can be reconstructed in the TPC to make fiducial volume cuts.

smaller for nuclear recoils (WIMPs, neutrons) than for electron recoils (electrons, gammas) making up most of the background.

Results from XENON10 The XENON Dark Matter Search program is a phased program: it aims to run a ton scale liquid Xenon (LXe) detector, but it proceeds step by step to gain experience from the operation of smaller detectors. The first Dark Matter Search step was the very successful operation of XENON10 at Laboratori Nazionali del Gran Sasso (LNGS), Italy, from 2005-2007. XENON10 was a $15 \mathrm{~kg}$ LXe detector with a fiducial mass of $5.4 \mathrm{~kg}$. A blind analysis of 58.8 live days, corresponding to an exposure of $136 \mathrm{~kg}$ days, lead to the best WIMP search limit in 2007, with a spin independent WIMP-nucleon cross section of $8.8 \times 10^{-44} \mathrm{~cm}^{2}$ at a WIMP mass of $100 \mathrm{GeV} / c^{2}$, and $4.5 \times 10^{-44} \mathrm{~cm}^{2}$ at $30 \mathrm{GeV} / c^{2}$ (90\% C.L.) [4].

The same data sample was also analyzed in terms of spin-dependent interactions [5]: this is possible since natural xenon contains about 50\% odd isotopes: $26.5 \%{ }^{129} \mathrm{Xe}$ ( $\operatorname{spin} 1 / 2$ ) and $21.2 \%{ }^{131} \mathrm{Xe}$ (spin 3/2). XENON10 excludes previously unexplored parameter space for neutralinos, where it is in particular sensitive to pure neutron couplings. Moreover, it excludes a heavy Majorana neutrino in the mass range of $9.4-2200 \mathrm{GeV} / c^{2}$ as a Dark Matter candidate.

XENON10 was limited by background since its materials were not chosen according to low intrinsic radioactivity. The main purpose of the experiment was to prove that this detector type works successfully and gives competitive results. XENON100, the next step in the phased program, is improved in terms of radioactivity and design.

\section{XENON100}

The aim of XENON100 is a sensitivity increase by a factor of $\sim 50$ compared to XENON10. This can be achieved by reducing the background in the fiducial volume by two orders of magnitude and increasing the detector mass by a factor of 10: it has a total mass of $170 \mathrm{~kg}$ LXe, where 70 $\mathrm{kg}$ are inside the TPC and the rest in an active veto region. We expect to have about $50 \mathrm{~kg}$ in the fiducial volume after geometric cuts.

The background reduction is achieved by a careful choice of materials and by using an improved detector design: all components with a known higher intrinsic radioactivity (cryogenics, feedthroughs) are now located outside the passive shielding. The shield of XENON10 was improved for this experiment: it now consists of $20 \mathrm{~cm}$ lead (inner $5 \mathrm{~cm}$ are low radioactivity lead, $17 \pm 5 \mathrm{~Bq} / \mathrm{kg}$ from $\left.{ }^{210} \mathrm{~Pb}\right), 20 \mathrm{~cm}$ of polyethylene, and an inner layer of up to $5 \mathrm{~cm}$ of copper. 


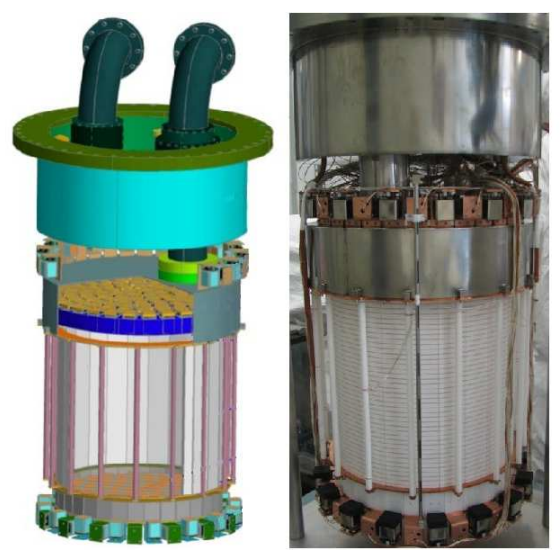

Figure 2: The XENON100 detector. The TPC contains $70 \mathrm{~kg}$ of LXe and is surrounded by an active LXe shield of $100 \mathrm{~kg}$. The detector is fully assembled and installed underground at LNGS since February 2008. The materials used for the detector were carefully chosen upon their low radioactivity and the detector design is such that all radioactive "hot" components are located far away from the sensitive volume, outside the shield.

Cryostat, cooling, and xenon purification The double wall cryostat is made out of low radioactivity stainless steel and has a weight of $70 \mathrm{~kg}$ only. Cooling is provided by a $170 \mathrm{~W}$ PTR cryocooler (Iwatani CryoMini Coldhead PC150) that liquefies the xenon gas outside the shield continuously.

In order to achieve high electron lifetimes in the liquid - a necessary requirement to drift charges over large distances - the number of electronegative impurities in the xenon has to be small. XENON100 uses a high temperature getter (SAES getters Mono Torr) to remove these impurities during constant xenon recirculation.

Xenon has no long lived isotopes that could give rise to intrinsic background. However, it contains a tiny fraction of radioactive ${ }^{85} \mathrm{Kr}\left(T_{1 / 2}=10.756 \mathrm{y}\right)$. In order to remove the krypton to the ppt level, the xenon gas was purified commercially and a sophisticated krypton distillation system (Taiyo Toyo Sanso Ltd.) was procured. This instrument allows us to purify the Xe from Kr on site.

TPC design, electric field configuration The XENON100 TPC is a cylinder with $15 \mathrm{~cm}$ radius and $30 \mathrm{~cm}$ height, made out of PTFE (teflon) to improve light collection (Fig. 2). The active volume is viewed by two PMT arrays: the top array consists of 98 PMTs arranged in concentric rings to improve radial fiducial volume cuts. 80 PMTs on the bottom array are placed as closely as possible to optimize light collection which finally determines the low energy threshold of the experiment. All light detectors are $1^{\prime \prime} \times 1^{\prime \prime}$ Hamamatsu R8520 low radioactivity PMTs. The bottom PMTs have an increased quantum efficiency of $\sim 33 \%$ with respect to the top array of PMTs.

A layer of LXe of about $3 \mathrm{~cm}$ thickness surrounds the whole TPC. This veto is made active with 64 more PMTs: $2 \times 16$ PMTs monitor the LXe above and below the target volume, respectively, and 32 PMTs look at the sides. We expect to gain an additional factor of $\sim 3-4$ in background reduction compared to using a passive veto.

The cathode at the lower end of the TPC is a $75 \mu \mathrm{m}$ thick stainless steel (SS) mesh in hexagonal geometry, the mesh pitch is $5 \mathrm{~mm}$. A cathode high voltage of $-30 \mathrm{kV}$ generates a drift field of $1 \mathrm{kV} / \mathrm{cm}$, corresponding to a maximal electron drift time of $\sim 160 \mu \mathrm{s}$. On the other side, the TPC is closed with a stack of 3 hexagonal SS meshes: the anode $(125 \mu \mathrm{m}, 2.5$ $\mathrm{mm}$ pitch) is located between two grounded meshes, the spacing is $5 \mathrm{~mm}$. An extraction field of $10 \mathrm{kV} / \mathrm{cm}$ is obtained by applying $+5 \mathrm{kV}$ to the anode. The whole stack is optimized for 
optical transparency and minimal impact on the $S 2$ energy resolution $(\sim 4 \%)$. 40 equidistant double ring field shaping rings are used to optimize the homogeneity of the drift field inside the TPC.

In order to ensure that the signal strength does not depend on the $x y$-position, the liquid gas interface has to be placed parallel to the anode. Four levelmeters measuring the capacitance of LXe filled SS tubes are used to level the TPC. The height of the liquid level can be adjusted since the anode stack is placed inside a "diving bell" with a gas outlet whose height can be changed by a motion feedthrough. The diving bell technique also allows to put a veto layer of xenon above the TPC and the top PMT array.

Data Acquisition and Slow Control system The XENON100 data acquisition system (DAQ) must be capable to digitize the full waveform of 242 PMTs, where the time window for an event is $320 \mu \mathrm{s}$. (This is the doubled maximal drift time to not miss an event when the DAQ triggered on $S 1$ instead of $S 2$.) At the same time, the maximal trigger rate has to be large enough to allow fast calibration.

We solved this issue by using CAEN V1724 flash ADC modules. This 14bit ADC with 10 ns time resolution has a circular buffer for a measurement without deadtime in Dark Matter mode. Additionally, it features an on-board FPGA used to decrease the data amount by removing the baseline between peaks which contains no interesting information: it only writes samples above an adjustable threshold into the output buffer and stores the time when the sample exceeded the threshold, thus the full waveform can be reconstructed. This allows to calibrate the detector at $\sim 400 \mathrm{~Hz}$ for $S 1$ pulses only, and with $\sim 50 \mathrm{~Hz}$ if $S 1$ and $S 2$ signals are measured simultaneously.

XENON100 is continuously monitored with a dedicated Slow Control system that records all relevant parameters of the experiment, such as temperatures (inside the cryostat in liquid and in gas, at the PTR, environment), pressures, flow rate (recirculation, filling, $\mathrm{N}_{2}$ purge), LXe levels, cryostat vacuum, Rn activity, high voltages, PMTs, etc.

\section{Expected backgrounds of XENON100}

For every dark matter experiment, an a-priori knowledge of its background has to be obtained in order to predict its sensitivity. This background can be mostly gammas, which produce electron recoils and neutrons, which produce nuclear recoils. A WIMP scattering off the target nucleus produces a nuclear recoil, therefore it cannot be distinguished from a neutron. The gamma background is reduced with an efficiency of $99.5 \%-99.9 \%$ [4] using the $S 2 / S 1$ ratio. Nevertheless it is crucial to minimize both kinds of background, in order to realize a factor of 100 reduction over XENON10, which had a gamma background of 0.6 dru in the WIMP search region. ( $1 \mathrm{dru}=1$ event $/ \mathrm{kg} / \mathrm{day} / \mathrm{keV}$, where $\mathrm{keV}$ is measured with the proper energy scale for nuclear recoils.) XENON100 achieves this by:

- placing hot materials, such as the cryogenics and the feedthroughs, outside the shielding. These materials were found to have a high radioactivity in XENON10, therefore they were moved far away from the detector;

- careful selection of ultra low background materials (c.f. Sec. 3.1);

- using $100 \mathrm{~kg}$ of LXe as an active shield. Events in the fiducial volume which are detected in coincidence with events in the veto will be rejected in the analysis. 


\section{THE XENON100 DARK MATter EXPERIMENT}

\subsection{Material screening}

The selection of most of the materials in XENON100 was done based on their low intrinsic radioactivity. XENON100 operates a dedicated screening facility underground at LNGS, consisting of a $2.2 \mathrm{~kg}$ high purity Ge crystal in an ultra-low background $\mathrm{Cu}$ cryostat, which is surrounded by a low background shielding $(\mathrm{Cu}$ and $\mathrm{Pb}$ ). Moreover, we also use the LNGS screening facility with some of the most sensitive Ge detectors in the world.

All the materials in XENON100 were screened in order to know their ${ }^{238} \mathrm{U},{ }^{235} \mathrm{U},{ }^{232} \mathrm{Th},{ }^{40} \mathrm{~K}$, and ${ }^{60} \mathrm{Co}$ contamination. Several low-activity materials were identified, such as the stainless steel used for the cryostat and the TPC. Table 1 shows the results from the screening of a subset of materials.

\begin{tabular}{|l|c|c|c|c|c|}
\hline Material & Unit & $\begin{array}{c}\mathrm{U} \\
{[\mathrm{mBq} / \mathrm{unit}]}\end{array}$ & $\begin{array}{c}\text { Th } \\
{[\mathrm{mBq} / \mathrm{unit}]}\end{array}$ & $\begin{array}{c}\mathrm{K} \\
{[\mathrm{mBq} / \mathrm{unit}]}\end{array}$ & $\begin{array}{c}\mathrm{Co} \\
{[\mathrm{mBq} / \mathrm{unit}]}\end{array}$ \\
\hline PTFE & $\mathrm{kg}$ & $<0.31$ & $<0.16$ & $<0.11$ & $\mathrm{NA}$ \\
Stainlees steel $(\mathrm{SS})$ & $\mathrm{kg}$ & $<1.9$ & $<1.0$ & $8.5 \pm 0.9$ & $10.5 \pm 4.2$ \\
PMT bases & $\mathrm{base}$ & $0.71 \pm 0.05$ & $0.10 \pm 0.03$ & $\mathrm{NA}$ & $\mathrm{NA}$ \\
22 high QE PMTs & $\mathrm{PMT}$ & $<0.24$ & $0.18 \pm 0.05$ & $11.5 \pm 2.0$ & $0.55 \pm 0.10$ \\
SS Screws for PMT bases & $\mathrm{kg}$ & $<9.2$ & $16 \pm 4$ & $9 \pm 3$ & $<46.4$ \\
Copper & $\mathrm{kg}$ & $<0.020$ & $<0.023$ & $\mathrm{NA}$ & $\mathrm{NA}$ \\
Polyethylene & $\mathrm{kg}$ & $<3.80$ & $<2.69$ & $<5.88$ & $<0.684$ \\
Outer $\mathrm{Pb}(516 \pm 90 \mathrm{~Bq} / \mathrm{kg})$ & $\mathrm{kg}$ & $<5.7$ & $<1.6$ & $<1.1$ & $14 \pm 6$ \\
Inner Pb $(17 \pm 5 \mathrm{~Bq} / \mathrm{kg})$ & $\mathrm{kg}$ & $<6.8$ & $<3.9$ & $<0.19$ & $<28$ \\
\hline
\end{tabular}

Table 1: A subset of materials screened with the Ge detectors of XENON100 and LNGS. The last four materials are used for the shielding of XENON100. The dominating activity for the two types of lead (given in the brackets) is due to ${ }^{210} \mathrm{~Pb}$.

\subsection{Gamma and neutron background studies}

The results from the material screening were used to predict the gamma and neutron induced background in XENON100. The geometry of the detector and its shielding was coded with the GEANT4 toolkit [6].

Gamma background Gammas from U, Th, K, and Co (and ${ }^{210} \mathrm{~Pb}$ for lead) were simulated for the detector and shielding materials. The rate of electron recoils which are generated in liquid xenon in the WIMP search region was estimated and is shown in Table 2. The detector position resolution (few $\mathrm{mm}$ ) and the activity of each material were taken into account. Fiducial volume cuts were applied to cut events at the edges of the detector, reducing the target mass to $\sim 50 \mathrm{~kg}$. The total rate of single scatters (WIMPs will never double scatter because of their low cross section) due to gammas is expected to be around 10 mdru before $S 2 / S 1$ discrimination, which is indeed about a factor of 100 lower than in XENON10.

Neutron background Neutrons are the most crucial background for many underground experiments. Experiments searching for WIMPs work with a very low energy threshold and are sensitive to - and should be protected from - neutrons from two possible sources: 


\begin{tabular}{|l|c|}
\hline Material & Single scatter rate $[\mathrm{mdru}]$ \\
\hline PTFE & $0.18 \pm 0.02$ \\
SS & $2.01 \pm 0.22$ \\
PMTs & $4.91 \pm 0.60$ \\
Copper & $0.026 \pm 0.002$ \\
Polyethylene & $2.50 \pm 0.29$ \\
Lead & negligible \\
\hline Total & $9.63 \pm 0.70$ \\
\hline
\end{tabular}

Table 2: Predicted gamma background rate in the WIMP search region $(4.5-26.9 \mathrm{keVnr})$. The differential rate unit is defined as $1 \mathrm{dru}=1$ event $/ \mathrm{kg} /$ day $/ \mathrm{keV}$.

1. Rock, detector, and shielding components, the so called local radioactivity: these neutrons have energies less than $10 \mathrm{MeV}$ and are produced via spontaneous fission and $(\alpha, \mathrm{n})$ reactions of ${ }^{238} \mathrm{U}$ and ${ }^{232} \mathrm{Th}$ on light elements.

2. Muons, the only cosmic-ray particles that can penetrate the rock down to hundreds of meters, produce high energy neutrons with a spectrum extending to $\mathrm{GeV}$ in spallations and electromagnetic and hadronic cascades. The neutron yield from cosmic-ray muons depends strongly on the depth of the underground laboratory [7].

Each of these background sources is studied separately in order to estimate the total neutron background of XENON100.

Radioactivity from detector and shielding materials: The local radioactivity from detector and shielding materials was calculated by generating the neutron spectra from spontaneous fission and $(\alpha, \mathrm{n})$ reactions following $\mathrm{U} / \mathrm{Th}$-decays and by propagating these neutrons into the fiducial volume. The neutron fluxes and the energy spectra from U/Th contamination in each material were calculated by the modified SOURCES4A code [8]. Figure 3 shows the neutron spectra from polyethylene (left) and $\mathrm{Cu}$ (right), assuming $10 \mathrm{ppb} \mathrm{U}$ contamination. It is obvious that $(\alpha, \mathrm{n})$ reactions contribute much less to the neutron spectrum of $\mathrm{Cu}$, since it is a high- $Z$ material.

Table 3 ( $2^{\text {nd }}$ column) shows the simulated neutron production rate for each material. These neutrons were propagated into the detector and the single scatter recoils in the fiducial volume
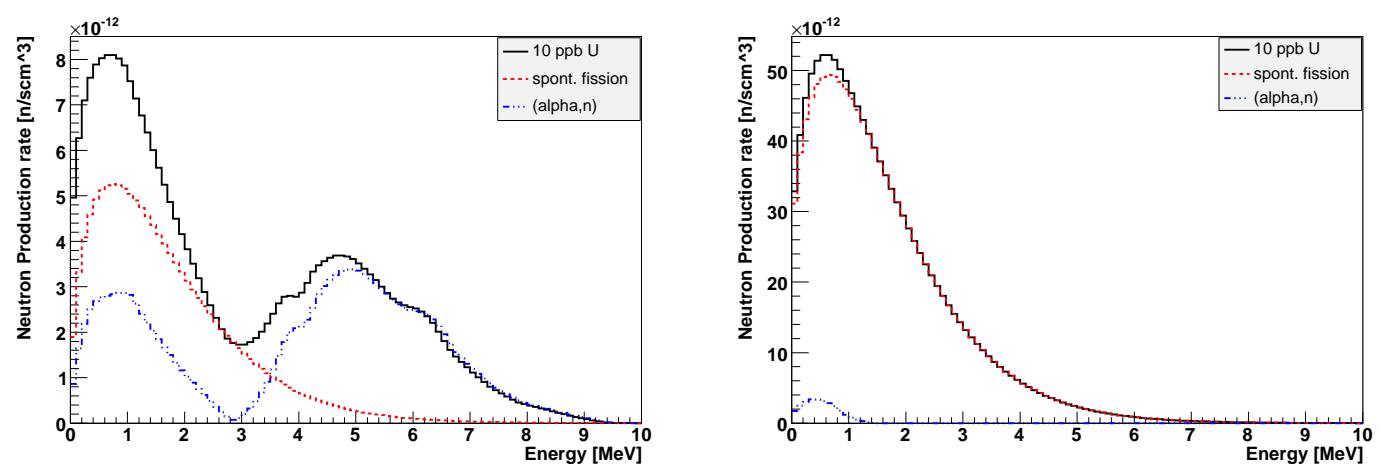

Figure 3: Neutron spectra from $10 \mathrm{ppb} \mathrm{U}$ in polyethylene (left) and in $\mathrm{Cu}$ (right). The dashed line shows the contribution from spontaneous fission, the dotted-dashed line from $(\alpha, \mathrm{n})$ reactions, and the solid line illustrates the total neutron production rate. 
THE XENON100 DARK MATTER EXPERIMENT

\begin{tabular}{|l|c|c|}
\hline Material & n/year & Single Scatters $\left[10^{-7} \mathrm{dru}\right]$ \\
\hline PTFE & 10.10 & $5.84 \pm 0.03$ \\
SS & 15.04 & $1.83 \pm 0.02$ \\
PMTs & 7.02 & $3.18 \pm 0.02$ \\
Copper & 1.58 & $0.11 \pm 0.002$ \\
Polyethylene & 416.26 & $4.89 \pm 0.10$ \\
Lead & 7384.56 & $0.38 \pm 0.02$ \\
\hline Total & - & $16.23 \pm 0.11$ \\
\hline
\end{tabular}

Table 3: Neutrons from detector materials: Column 2: Neutrons produced in detector and shielding material per year. Column 3: Mean rate of single scatter nuclear recoils in the WIMP search region $(4.5-26.9 \mathrm{keVnr})$.

were recorded as shown in Fig. 4. Table 3 ( $3^{\text {rd }}$ column) also gives the mean single scatter nuclear recoil rate in the WIMP search region. The fraction of single recoils is $\sim 44 \%$. The total rate of nuclear single scatters from local radioactivity is estimated to be $1.6 \mu \mathrm{dru}$, which corresponds to 0.6 single nuclear recoils per year in the fiducial volume.

Radioactivity from the cavern: The neutron background from the experimental environment is calculated in a similar way as above. The cavern in which the XENON100 detector is located is simulated to consist of $30 \mathrm{~cm}$ concrete followed by rock. Material composition and activities were taken from [10]. Since the detector is located close to LNGS Hall A, the same activity values were assumed for the XENON100 cavern. The rock thickness is considered to be $3 \mathrm{~m}$, since no neutrons from larger distances can reach the rock-cavern boundary. Using the SOURCES4A code, the neutron production rate was calculated to be 1.7 and 7.1 per year per gram from concrete and rock, respectively. Even if the production rate from rock is higher, the dominant contribution is expected to be from concrete, which reduces the neutron flux from the rock significantly. These simulations, however, are still in progress.

Event rate from cosmic-ray muons: The muon transportation through the Gran Sasso rock down to a depth of 3600 m.w.e. was done with the MUSIC code [7]. Muon energy and angular distribution were calculated with MUSUN [7]. The mean muon energy, which increases with

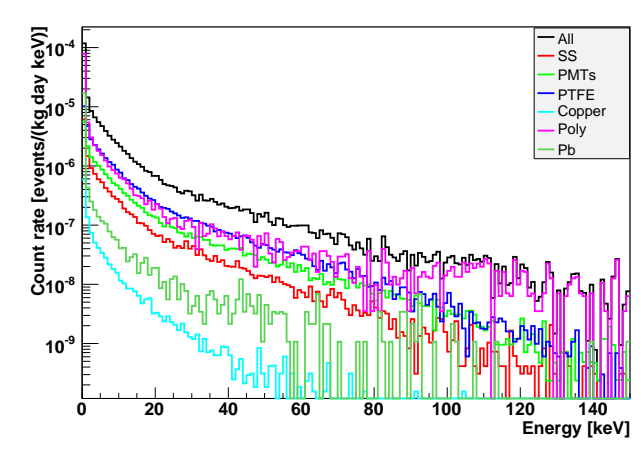

Figure 4: Single scatter nuclear recoils in the fiducial volume of XENON100 due to U/Th contamination in the detector and shielding materials. The spectra correspond to the total rate generated in PTFE, polyethylene, PMTs, $\mathrm{SS}, \mathrm{Pb}, \mathrm{Cu}$, and LXe (top to bottom).

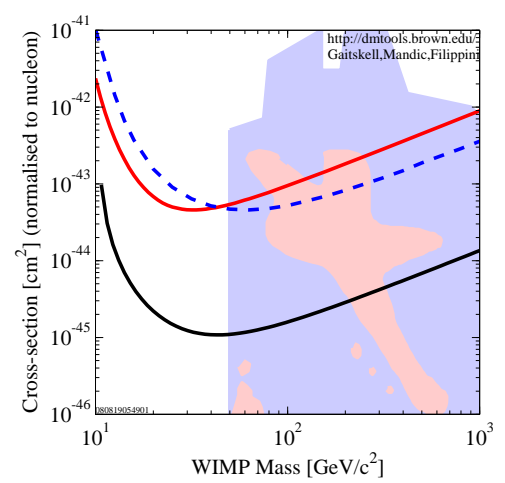

Figure 5: Projected sensitivity of XENON100 (black), reaching a sensitivity of $2 \cdot 10^{-45} \mathrm{~cm}^{2}$ for a $100 \mathrm{GeV}$ WIMP. Additionally, the current limits of XENON10 (red) [4] and CDMS (dashed blue) [9] are shown. 
the lab depth, is $270 \mathrm{GeV}$ in LNGS, and the muon flux is $1.17 \mathrm{~m}^{-2} \mathrm{~h}^{-1}$. For this simulation, the rock thickness is assumed to be $6 \mathrm{~m}$, which is a sufficient distance for all the hadronic cascades to develop and to produce neutrons. These neutrons then were propagated into the detector. It is known that neutron production from muons is more favored in high- $Z$ materials [7], therefore many neutrons will be produced inside the shielding. These simulation are in progress and final results are not available yet, but it is expected to be a sub-dominant neutron background compared to the local radioactivity.

\section{Summary and outlook}

The XENON100 detector is installed underground at LNGS, Italy, in a depth of $3600 \mathrm{~m}$ water equivalent since February 2008. Since then, almost all components have been tested successfully and calibration and background data are taken continuously in order to characterize the response and performance of the experiment. The first Dark Matter run is scheduled for the end of 2008 .

Based on the detailed background studies using the measured activities of the detector components, we expect a gamma background of about 0.01 events $/ \mathrm{keVee} / \mathrm{kg} /$ day and less than 0.6 neutrons/year (from local radioactivity) in the fiducial volume and the WIMP search region. Assuming the same energy threshold as achieved in XENON10 and the same electron recoil rejection power, we will be able to run XENON100 background free for about 2 months with $\sim 50 \mathrm{~kg}$ fiducial mass, reaching a sensitivity of $2 \cdot 10^{-45} \mathrm{~cm}^{2}$ for a $100 \mathrm{GeV}$ WIMP (Fig. 5).

At the same time, the XENON collaboration already started to study the design and the backgrounds of a larger LXe detector which will probe a large fraction of the predicted WIMP parameter space.

\section{References}

[1] W.-M. Yao et al. (PDG), Journ. of Phys. G33 1 (2006) and references therein.

[2] A. Bottino et al., Phys. Rev. D69 037302 (2004);

J. Ellis et al., Phys. Rev. D71 095007 (2005);

H. C. Cheng et al., Phys. Rev. Lett. 89211301 (2002);

A. Birkedal-Hansen and J. G. Wacker, Phys. Rev. G69 065022 (2004).

[3] E. Aprile et al., New Astron. Rev. 49289 (2005).

[4] J. Angle et al. (XENON10), Phys. Rev. Lett. 100021303 (2008).

[5] J. Angle et al. (XENON10), Phys. Rev. Lett. 101091301 (2008).

[6] S. Agostinelli et al., Nucl. Instr. Meth. A506 250 (2003).

[7] P. Antonioli et al., Astrop. Physics 7357 (1997);

V. A. Kudryavtsev et al., Phys. Lett. B471 251 (1999);

V. A. Kudryavtsev et al., Nucl. Instr. Meth. A505 688 (2003).

[8] Wilson et al, Phys. Lett. B645 153 (2007);

M. Carson et al, Astrop. Physics 21667 (2004);

R. Lemrani et al, Nucl. Instr. Meth. A560 454 (2006);

V. Tomasello et al, doi:10.1016/j.physletb.2003.10.071.

[9] Z. Ahmed et al. (CDMS), arXiv:0802.3530 (2008).

[10] H. Wulandari et al., Astrop. Physics 22313 (2004). 